\title{
STABILITY OF POLE SOLUTIONS FOR PLANAR PROPAGATING FLAMES: I. EXACT EIGENVALUES AND EIGENFUNCTIONS*
}

\author{
DIMITRI VAYNBLAT ${ }^{\dagger}$ AND MOSHE MATALON ${ }^{\dagger}$
}

\begin{abstract}
It is well known that the nonlinear PDE describing the dynamics of a hydrodynamically unstable planar flame front admits exact pole solutions as equilibrium states. Such a solution corresponds to a steadily propagating cusp-like structure commonly observed in experiments. In this work we investigate the linear stability of these equilibrium states - the steady coalescent pole solutions. In previous similar studies, either a truncated linear system was numerically solved for the eigenvalues or the initial value problem for the linearized PDE was numerically integrated in order to examine the evolution of initially small disturbances in time. In contrast, our results are based on the exact analytical expressions for the eigenvalues and corresponding eigenfunctions. In this paper we derive the expressions for the eigenvalues and eigenfunctions. Their properties and the implication on the stability of pole solutions is discussed in a paper which will appear later.
\end{abstract}

Key words. flame front, pole decomposition, coalescent pole solution, stability, eigenvalues and eigenfunctions

AMS subject classifications. 76E99, 35Q35, 80A32

PII. S0036139998346439

1. Introduction. In the context of a weak thermal expansion approximation, Sivashinsky [1] derived a single PDE that describes the nonlinear evolution of a planar flame front subjected to the Darrieus-Landau instability $[2,3]$. When periodic boundary conditions are assumed, the problem can be expressed in terms of a single parameter $\gamma$ which is proportional to the size of the domain of integration $L$ and inversely proportional to the gas thermal expansion. A trivial solution of this equation, corresponding to a planar flame front, is known to be unstable for $\gamma>\gamma_{1}=2$. Numerical integration of the nonlinear PDE confirms this prediction. For $\gamma<\gamma_{1}$, regardless of the initial conditions, the solution after a sufficiently long time always converges to the trivial solution. For $\gamma>\gamma_{1}$, however, the initial perturbations intensify and the solution rapidly diverges away from the flat front.

The evolution of the flame front beyond the stability threshold has been examined by various authors, see, for example, $[4,5,6,7,8,9]$. In a typical numerical experiment, one first observes the development of several wrinkles along the flame front which eventually coalesce into one large peak as time progresses. After sufficiently long time, the cusp-like structure appears to propagate at a constant speed without change in shape. The structure of the solution is retained as $\gamma$ increases, except that now more wrinkles are formed initially and the depth of the peak that eventually forms, intensifies, and approaches a real cusp as $\gamma \rightarrow \infty$. Some numerical experiments [5, 9], however, suggest that for sufficiently large values of $\gamma$ the equation displays a qualitatively different behavior: new cusp-like structures appear repetitively on the flame front and the speed of propagation increases indefinitely. It has been tempting to associate this peculiar behavior with the development of sec-

*Received by the editors October 29, 1998; accepted for publication March 23, 1999; published electronically February 2, 2000. This research was partially supported by the National Science Foundation under grants DMS9703716 and CTS9521022.

http://www.siam.org/journals/siap/60-2/34643.html

${ }^{\dagger}$ Department of Engineering Sciences and Applied Mathematics, McCormick School of Engineering and Applied Science, Northwestern University, Evanston, IL 60208-3125 (matalon@nwu.edu).

${ }^{\ddagger}$ Current address: Applied Mathematics 217-50, Caltech, Pasadena, CA 91125. 
ondary structures which have been observed experimentally on the propagating fronts of sufficiently large flames $[10,11]$.

Whether the unsteady behavior uncovered numerically for large values of $\gamma$ is indeed inherent to the (one-dimensional) nonlinear PDE or is a mere product of computational noise is a question that has been debated in the literature. What has prompted this discussion is the fact that the nonlinear PDE admits exact equilibrium solutions obtained by a pole decomposition technique and called coalescent pole solutions. Large peak solutions emerging when the nonlinear PDE is numerically integrated over a long time appear to belong to the family of these solutions. The nonsteady behavior of the solution of the PDE, presumably observed for large values of $\gamma$, does not seem to agree with the expectation from the pole-decomposition theory which does not distinguish between small and large values of $\gamma$. Joulin and coworkers $[6,12]$ argue that the PDE is therefore not capable of describing the repetitive generation of new "cusps" when $\gamma$ is large. The appearance of new "cusps" in the computations $[5,9]$ results from the limitations of the numerics. To describe mathematically the experimental observation, specific to large flames [10,11], new models need to be derived. Sivashinsky and co-workers $[7,8]$, on the other hand, argue that the inconsistencies with the pole-decomposition theory lie in the stability of the exact pole solutions. The nonsteady behavior may be associated with the fact that these equilibrium solutions are unstable when $\gamma$ is sufficiently large. By numerically solving the initial value problem that results from linearizing the PDE about a pole solution they concluded that, for large values of $\gamma$, pole solutions are unstable. The linear stability of pole solutions was also addressed in $[13,14]$ using a different approach. The eigenvalue problem for the perturbed system was formulated and the eigenvalues of the corresponding truncated matrix were determined numerically. In contrast to the results of $[7,8]$ it was concluded that, for any value of $\gamma$, there exists a stable pole solution. Their conclusion thus supports Joulin's view on this matter.

In this study we also address the linear stability of pole solutions. However, in contrast to the previous studies, we construct exact analytical expressions for the eigenvalues and eigenfunctions. Based on these expressions we make definite statements about the stability question which resolve unequivocally the controversies discussed above. We show that for any value of $\gamma$ there exists a stable pole solution. Therefore, the conclusions of $[13,14]$ and correspondingly the arguments presented in $[6,12]$ agree with our results. (The nature of the spectrum deduced numerically in $[13,14]$, however, is not accurate). The conclusions drawn in $[7,8]$, on the other hand, do not agree with our exact results. We believe that the erroneous conclusions reported in $[7,8]$, namely, that the equilibrium pole solutions become unstable when $\gamma$ is sufficiently large, result from the relatively low number of modes included in their numerical calculations for the relatively large values of $\gamma$ considered.

The work is the first of two papers. In this paper we introduce the nonlinear evolution equation, review some of the relevant material about pole solutions, and derive the analytic expressions for the eigenvalues and the eigenfunctions. In a paper that will appear later [15], which we refer to as Part II, we present the properties of the eigenvalues and the eigenfunctions and discuss the implications on the stability of pole solutions and on the nonlinear PDE describing the evolution of flame front perturbations on a nominally planar front. Our conclusions, summarized above, are discussed in more detail in [15].

This paper is organized as follows. In section 2, we introduce the nonlinear evolution equation and comment on some of its general properties. In section 3 , we 
review pole solutions; a detailed consideration is given to the family of the coalescent steady states. In section 4, we derive analytic expressions for the eigenvalues and the eigenfunctions. In section 5, we summarize the results and present an outline of Part II.

2. The evolution equation. In the one-dimensional case and in a dimensionless form, the nonlinear PDE that describes the dynamics of a planar flame front subject to Darrieus-Landau instability [1] can be written as

$$
\varphi_{t}=\frac{1}{2} I\{\varphi ; x\}+\alpha \varphi_{x x}+\frac{1}{2} \varphi_{x}^{2} .
$$

Here $\varphi(x, t)$ is the flame front displacement, $x$ is the spatial coordinate, $t$ is the time, and $\alpha$ is a parameter that depends on the gas thermal expansion. Distance and time are made dimensionless using $l_{D}$ and $l_{D} / S_{L}$ as units, respectively; $l_{D}$ is the diffusion length and $S_{L}$ is the laminar flame speed. The operator $I\{\cdot ; x\}$ is a linear singular nonlocal operator defined such that $I\{\varphi ; x\}=-\mathcal{H}\left\{\varphi_{x} ; x\right\}$, where $\mathcal{H}\{\cdot ; x\}$ is the Hilbert transform. Equation (2.1) is to be integrated over the interval $-L \leq x \leq L$, and periodic boundary conditions are assumed. It should be pointed out that the evolution equation could be written in slightly different forms $[1,8,14]$ that are easily obtained by rescaling (2.1).

It is worth mentioning that the operator $I\{\cdot ; x\}$ can also be defined in terms of the spatial Fourier transform. Let

$$
\varphi(x, t)=\sum_{k=-\infty}^{\infty} \hat{\varphi}_{k}(t) e^{i \tilde{k} x}
$$

then $I: \hat{\varphi}_{k}(t) \mapsto|\tilde{k}| \hat{\varphi}_{k}(t)$, where $\tilde{k}=\pi k / L$ is the spatial wave number, with $k$ an integer. Then in physical space the operator $I\{\cdot ; x\}$ can be written as

$$
I\{\varphi ; x\}=\frac{1}{L} \sum_{k=1}^{\infty} \tilde{k} \int_{-L}^{L} \cos [\tilde{k}(x-\eta)] \varphi(\eta, t) d \eta .
$$

Throughout this paper we will be using the convention that an accent "tilde" represents a multiplication by $\pi / L$.

Although the problem contains two parameters, $\alpha$ and $L$, it can be reduced to a single parameter problem $[16,14,7]$ by rescaling the variables as $\tilde{x}=\pi x / L, \tilde{t}=\pi t / L$, and $\tilde{\varphi}(\tilde{x}, \tilde{t})=\pi \varphi(x, t) / L$. Such a transformation leads to rescaling the length $2 L$ of the domain so that it always takes the fixed value $2 \pi$. The scaled equation is now

$$
\tilde{\varphi}_{\tilde{t}}=\frac{1}{2} I\{\tilde{\varphi} ; \tilde{x}\}+\tilde{\alpha} \tilde{\varphi}_{\tilde{x} \tilde{x}}+\frac{1}{2} \tilde{\varphi}_{\tilde{x}}^{2}
$$

which is to be integrated over the interval $-\pi \leq \tilde{x} \leq \pi$, with $\tilde{\alpha}=\pi \alpha / L$ the only parameter. We also introduce $\gamma=1 / \tilde{\alpha}$ which turns out to be the more suitable parameter to use when expressing the results. Both $\tilde{\alpha}$ and $\gamma$, however, will be used interchangeably in the following. We note that changes in $L$, the parameter used in $[7,13,14,8]$, are proportional to changes in $\gamma$ and inversely proportional to changes in $\tilde{\alpha}$.

As pointed out in [16], (2.4) has a scaling invariance: If $\tilde{\varphi}_{1}(\tilde{x}, \tilde{t})$ is a solution of (2.4) on the interval $0 \leq \tilde{x} \leq 2 \pi$ for $\tilde{\alpha}_{1}$, then, given a positive integer $n, \tilde{\varphi}_{n}(\tilde{x}, \tilde{t})=$ $\frac{1}{n} \tilde{\varphi}_{1}(n \tilde{x}, n \tilde{t})$ is a solution on the same interval for $\tilde{\alpha}_{n}=\frac{1}{n} \tilde{\alpha}_{1}$. 
In the discussion below, we found it more convenient to use the slope of the flame front rather than the displacement. The change of variables $u(x, t)=\varphi_{x}(x, t)$ in $(2.1)$ leads to the following PDE:

$$
u_{t}=\frac{1}{2} I\{u ; x\}+\alpha u_{x x}+\left(\frac{1}{2} u^{2}\right)_{x}
$$

In Fourier space, (2.5) takes the form

$$
\left(\hat{u}_{k}\right)_{t}=\frac{1}{2}|\tilde{k}| \hat{u}_{k}-\alpha \tilde{k}^{2} \hat{u}_{k}+\left[\left(\widehat{\frac{1}{2} u^{2}}\right)_{x}\right]_{k}, \quad k=-\infty, \ldots,-1,1, \ldots, \infty
$$

where $\hat{u}_{k}(t)=i \tilde{k} \hat{\varphi}_{k}(t)$ and $\hat{\varphi}_{k}(t)$ is defined in (2.2).

3. Pole solutions. Thual, Frisch, and Hénon [17] recognized that (2.5) corresponds to a more general class of equations, arising in plasma physics [18], for which an infinite number of exact solutions exists. An $N$-pole solution of (2.5) is of the form

$$
u(x, t)=\alpha \tilde{r} \sum_{n=1}^{N}\left[\cot \left(\frac{\tilde{r} x-z_{n}(t)}{2}\right)+\cot \left(\frac{\tilde{r} x-z_{n}^{*}(t)}{2}\right)\right]
$$

where $N$, an integer, is the number of poles $z_{n}(t)$ in the complex plane; $\tilde{r}=\pi r / L$, with $r$ an integer, is the wavenumber; and the asterisk denotes the complex conjugate. Writing

$$
z_{n}(t) \equiv x_{n}(t)+i y_{n}(t), \quad n=1, \ldots, N
$$

the solution can also be written as

$$
u(x, t)=2 \alpha \tilde{r} \sum_{n=1}^{N} \frac{\sin \left(\tilde{r} x-x_{n}(t)\right)}{\cosh \left(y_{n}(t)\right)-\cos \left(\tilde{r} x-x_{n}(t)\right)} .
$$

In terms of the flame displacement, the solution takes form

$$
\begin{aligned}
\varphi(x, t) & =\varphi_{0}(t)+2 \alpha \sum_{n=1}^{N}\left\{\ln \left[\sin \left(\frac{\tilde{r} x-z_{n}(t)}{2}\right)\right]+\ln \left[\sin \left(\frac{\tilde{r} x-z_{n}^{*}(t)}{2}\right)\right]\right\} \\
& =\varphi_{0}(t)+2 \alpha \sum_{n=1}^{N} \ln \frac{1}{2}\left[\cosh \left(y_{n}(t)\right)-\cos \left(\tilde{r} x-x_{n}(t)\right)\right]
\end{aligned}
$$

where $\varphi_{0}(t)$ is a linear function of time such that

$$
\dot{\varphi}_{0}=N \alpha \tilde{r}(1-2 N \alpha \tilde{r})
$$

with the dot denoting differentiation with respect to time. Note that for $u(x, t)$ and $\varphi(x, t)$ to be real-valued functions, the $N$ poles should appear in pairs of complex conjugates.

A pole in the complex plane corresponds, in the physical plane, to a "cusp" rounded at its tip (to which we shall refer loosely as a cusp). Hence, the solution (3.4) is a superposition of a finite number of cusps. The real part of a pole, $x_{n}(t)$, which is always in the interval $[-\pi,+\pi]$, corresponds to the location of the cusp along the flame 
front; the imaginary part of the pole, $y_{n}(t)$, determines the depth of the cusp. As $y_{n}(t)$ decreases, the cusp becomes deeper and its depth tends to infinity as $y_{n}(t) \rightarrow 0$; in the limit one obtains a real cusp. On the other hand, the depth tends to zero as $y_{n}(t) \rightarrow \infty$. Finally, note that the solutions for different values of $r$ are simply the images of the solution for $r=1$ under the scaling transformation mentioned in section 2 (see also (3.5), and (3.6) below).

Substituting (3.3) in the PDE (2.5) results in a system of ODEs that govern the dynamics of the poles in the complex plane. One finds

$$
\frac{\dot{z}_{n}}{\tilde{r}}=-\alpha \tilde{r} \sum_{\substack{l=1 \\ l \neq n}}^{2 N} \cot \left(\frac{z_{n}-z_{l}}{2}\right)-i \frac{1}{2} \operatorname{sgn}\left[\Im\left(z_{n}\right)\right], \quad n=1, \ldots, 2 N,
$$

where $\Im$ denotes the imaginary part, and the signum function is defined, as usual, by $\operatorname{sgn}(x)=|x| / x$ for $x \neq 0$, and $\operatorname{sgn}(0)=0$. Expressing these equations in terms of their real and imaginary parts (because the poles appear in pairs of complex conjugates, it is sufficient to write the equation for only positive $y_{n}$ ) results in

$$
\begin{aligned}
\frac{\dot{x}_{n}}{\tilde{r}} & =-\alpha \tilde{r} \sum_{\substack{l=1 \\
l \neq n}}^{N} \sin \left(x_{n}-x_{l}\right)\left(\left[\cosh \left(y_{n}-y_{l}\right)-\cos \left(x_{n}-x_{l}\right)\right]^{-1}\right. \\
& \left.+\left[\cosh \left(y_{n}+y_{l}\right)-\cos \left(x_{n}-x_{l}\right)\right]^{-1}\right), \quad n=1, \ldots, N, \\
\frac{\dot{y}_{n}}{\tilde{r}} & =\alpha \tilde{r} \sum_{\substack{l=1 \\
l \neq n}}^{N}\left(\frac{\sinh \left(y_{n}-y_{l}\right)}{\cosh \left(y_{n}-y_{l}\right)-\cos \left(x_{n}-x_{l}\right)}+\frac{\sinh \left(y_{n}+y_{l}\right)}{\cosh \left(y_{n}+y_{l}\right)-\cos \left(x_{n}-x_{l}\right)}\right) \\
& +\alpha \tilde{r} \operatorname{coth}\left(y_{n}\right)-\frac{1}{2}, \quad n=1, \ldots, N .
\end{aligned}
$$

Throughout this paper, we found it convenient to use the Fourier transform of the pole solution (3.3). The correspondence is obtained by using

$$
u(x, t)=\sum_{\substack{m=-\infty \\ m \neq 0}}^{\infty} \hat{u}_{m}(t) e^{i \tilde{m} x}
$$

where

$$
\hat{u}_{m}(t)= \begin{cases}-2 i \tilde{\alpha} r \operatorname{sgn}(k) \sum_{n=1}^{N} e^{-i k x_{n}(t)-|k| y_{n}(t)} & \text { if } m=k r \\ 0 & \text { otherwise. }\end{cases}
$$

It should be noticed that all solutions of the PDE (2.1) correspond to flame fronts propagating, on the whole, upwards; i.e., in the direction of $\varphi>0$. This is easily seen by taking the spatial average of the equation over a period of $2 L$. Defining $\langle\psi\rangle=\frac{1}{2 L} \int_{-L}^{L} \psi(x) d x$, one finds that

$$
\langle\varphi\rangle_{t}=\frac{1}{2}\left\langle\left(\varphi_{x}\right)^{2}\right\rangle
$$


is always positive. For the pole solution (3.4) this implies that

$$
\langle\varphi\rangle_{t}=\dot{\varphi}_{0}+2 \alpha \sum_{n=1}^{N} \dot{y}_{n}(t) \geq 0
$$

If we now consider a steady pole solution (for which all the poles are time independent), it immediately follows from (3.12) that the constant $\dot{\varphi}_{0}$ is positive. Therefore, (3.5) implies that, for a given $\alpha \tilde{r}$, the number of poles is bounded, $N \leq N_{\max }$. The upper bound is given by

$$
N_{\max }=\operatorname{Int}\left[\frac{1}{2 \alpha \tilde{r}}\right],
$$

where $\operatorname{Int}(x)$ denotes the greatest integer less than or equal to the real number $x$.

3.1. Coalescent pole solution. A particular set of pole solutions of (2.5) corresponds to the poles being aligned parallel to the imaginary axis. Thual, Frisch, and Hénon [17] noticed that there exists a natural tendency for the poles to align themselves in this way. Their argument is based on an observation of (3.7)-(3.8). In the horizontal direction the poles attract each other since the sign of $\dot{x}_{n}$ is always determined by that of $\sin \left(x_{n}-x_{l}\right)$. In the vertical direction, in addition to being drifted toward the real axis, the poles repel each other at short range and attract each other at long range. Thus the poles eventually align themselves vertically and coalesce into a single line. Following [7], we shall refer to this solution as a coalescent solution.

For a coalescent solution, $\dot{x}_{n}=0, \quad n=1, \ldots, N$. The set of ODEs (3.7) is always satisfied, while the set of ODEs (3.8) becomes

$$
\frac{\dot{y}_{n}}{\tilde{r}}=\alpha \tilde{r} F_{n}\left(y_{1}, \ldots, y_{N}\right), \quad n=1, \ldots, N,
$$

where

$$
F_{n}\left(y_{1}, \ldots, y_{N}\right)=\sum_{\substack{l=1 \\ l \neq n}}^{N}\left[\operatorname{coth}\left(\frac{y_{n}-y_{l}}{2}\right)+\operatorname{coth}\left(\frac{y_{n}+y_{l}}{2}\right)\right]+\operatorname{coth}\left(y_{n}\right)-\frac{1}{2 \alpha \tilde{r}} .
$$

Without loss of generality we assume that the $y_{n}$ 's have been ordered such that $0<y_{1}<\cdots<y_{N}$. We note that this order does not change during the evolution since the repulsion between two poles becomes infinite when they approach each other. For the same reason, we always have strict inequalities in this ordering.

In physical and Fourier spaces, the coalescent pole solution is of the form (from (3.3), (3.4), and (3.10))

$$
\begin{gathered}
u(x, t)=2 \alpha \tilde{r} \sum_{n=1}^{N} \frac{\sin \left(\tilde{r} x-x_{c}\right)}{\cosh \left(y_{n}(t)\right)-\cos \left(\tilde{r} x-x_{c}\right)}, \\
\varphi(x, t)=\varphi_{0}(t)+2 \alpha \sum_{n=1}^{N} \ln \frac{1}{2}\left[\cosh \left(y_{n}(t)\right)-\cos \left(\tilde{r} x-x_{c}\right)\right],
\end{gathered}
$$


and

$$
\hat{u}_{m}(t)= \begin{cases}-2 i \tilde{\alpha} r \operatorname{sgn}(k) e^{-i k x_{c}} \sum_{n=1}^{N} e^{-|k| y_{n}(t)} & \text { if } m=k r \\ 0 & \text { otherwise }\end{cases}
$$

where $x_{c}$ is the real (common) part of the coalescent poles. For future reference, we also introduce the shifted sine transform of the slope of the flame front

$$
u(x, t)=\sum_{m=1}^{\infty} \hat{u}_{m}^{\sin }(t) \sin \left[m\left(\tilde{x}-\frac{x_{c}}{r}\right)\right],
$$

where

$$
\hat{u}_{m}^{\sin }(t)= \begin{cases}4 \tilde{\alpha} r \sum_{n=1}^{N} e^{-k y_{n}(t)} & \text { if } m=k r \\ 0 & \text { otherwise. }\end{cases}
$$

The Fourier transform of the slope, $\hat{u}_{m}$, can be easily expressed in terms of the shifted sine transform, namely,

$$
\hat{u}_{m}(t)=-i \operatorname{sgn}(m) \frac{1}{2} e^{-i k x_{c}} \hat{u}_{|m|}^{\sin }(t) .
$$

3.2. Steady coalescent pole solution. A coalescent solution for which all the poles are time independent is referred to as a coalescent steady state [7]. A coalescent steady state is a solution of $N$ simultaneous nonlinear equations (see (3.14)):

$$
F_{n}\left(y_{1}, \ldots, y_{N}\right)=0, \quad n=1, \ldots, N .
$$

The last equation of (3.22), corresponding to $n=N$ (see also (3.15)), together with the fact that $\operatorname{coth}(x)>1$ for $x>0$, yields the inequality

$$
\tilde{\alpha}<\frac{1}{2 r(2 N-1)} \quad \text { or } \quad \gamma>2 r(2 N-1) .
$$

This implies that, for a given $\alpha \tilde{r}$, there exist coalescent steady states only if the number of poles satisfies the inequality $N \leq N_{0}$, with $N_{0}$ given by

$$
N_{0}= \begin{cases}\operatorname{Int}\left[\frac{1}{4 \alpha \tilde{r}}+\frac{1}{2}\right] & \text { if } \frac{1}{4 \alpha \tilde{r}}+\frac{1}{2} \text { is not an integer, } \\ \operatorname{Int}\left[\frac{1}{4 \alpha \tilde{r}}-\frac{1}{2}\right] & \text { otherwise. }\end{cases}
$$

Thual, Frisch, and Hénon [17] proved that the following two properties hold for $N \leq N_{0}$ : (i) there exists one and only one coalescent steady state, and (ii) any solution of (3.14) tends towards that steady state as $t \rightarrow \infty$. This means that coalescent steady states are stable solutions of the system (3.14), i.e., when only coalescent solutions are considered. Whether these states are also stable solutions of the full set of ODEs (3.6) and, what is more important, stable solutions of the PDE (2.1), remains to be determined. This question, which is the main objective of this work, will be discussed in the following section. 


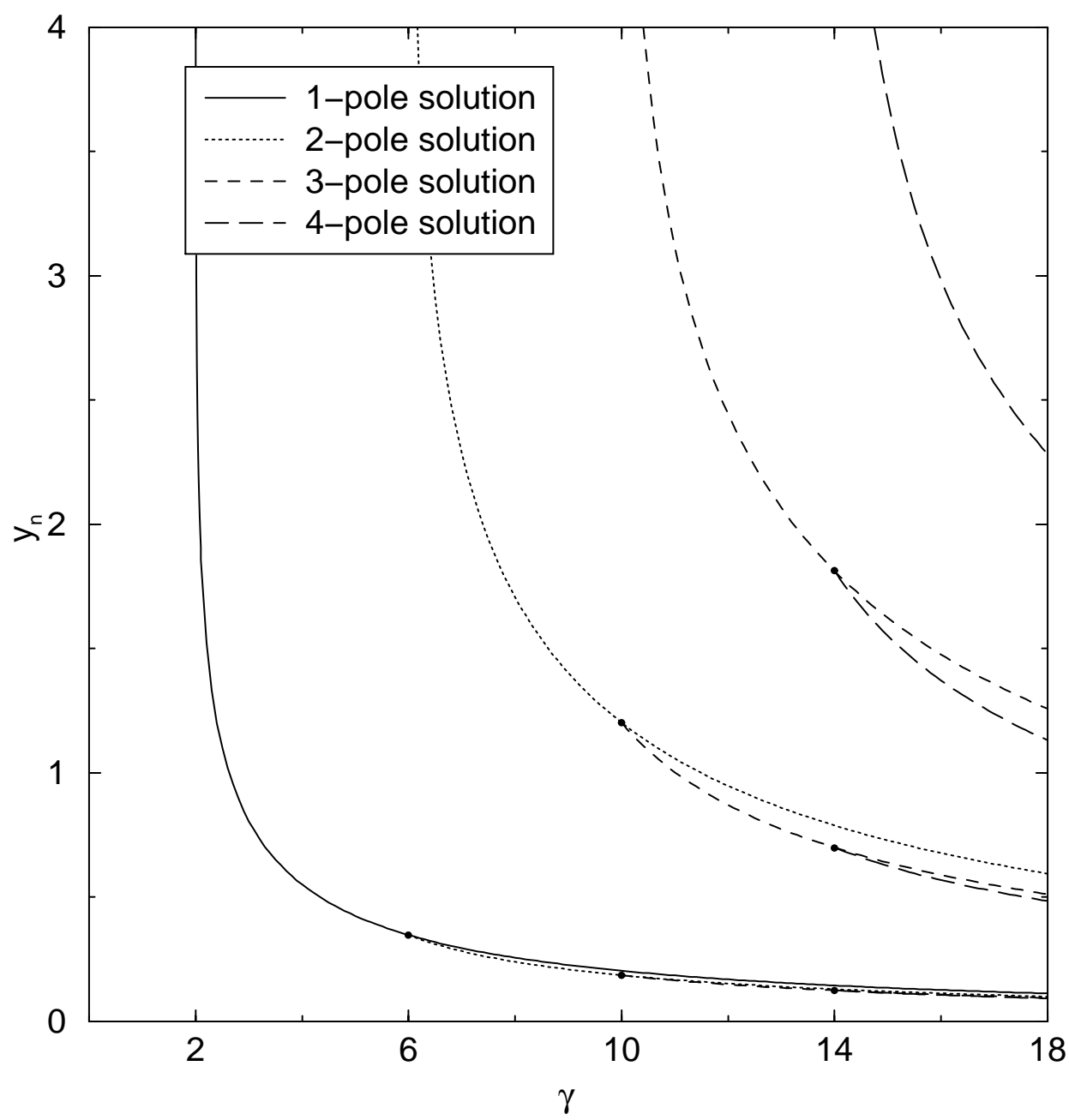

FIG. 3.1. The dependence of the imaginary parts of poles, $y_{n}$, on $\gamma$ for $N$-pole coalescent steady states, $N=1, \ldots, 4$. The points where the new poles are born are marked with dots.

The solutions of (3.22) have simple analytical forms for $N=1,2$. For $N=1$,

$$
y_{1}=\tanh ^{-1}(2 \alpha \tilde{r}) .
$$

For $N=2$,

$$
y_{1,2}=\tanh ^{-1}(6 \alpha \tilde{r}) \mp \frac{1}{2} \cosh ^{-1}\left(\frac{1}{1-(6 \alpha \tilde{r})^{2}}\right) .
$$

For $N>2,(3.22)$ must be solved numerically. This has been carried out using a Newton-Raphson method [19]. The position $y_{n}$ of each pole is adjusted to a new value

$$
y_{n}^{\text {new }}=y_{n}^{\text {old }}+\delta y_{n}, \quad n=1, \ldots, N,
$$


with the correction vector $\delta y_{n}$ obtained by solving the matrix equation

$$
\sum_{j=1}^{N} \frac{\partial F_{i}}{\partial y_{j}} \delta y_{j}=-F_{i}, \quad i=1, \ldots, N,
$$

where the entries of the Jacobian matrix are given by

$$
\begin{aligned}
& \frac{\partial F_{i}}{\partial y_{j}}=\frac{1}{2}\left[\left(\sinh \left[\frac{y_{i}-y_{j}}{2}\right]\right)^{-2}-\left(\sinh \left[\frac{y_{i}+y_{j}}{2}\right]\right)^{-2}\right], \quad j \neq i, \\
& \frac{\partial F_{i}}{\partial y_{i}}=-\frac{1}{2} \sum_{\substack{j=1 \\
j \neq i}}^{N}\left[\left(\sinh \left[\frac{y_{i}-y_{j}}{2}\right]\right)^{-2}+\left(\sinh \left[\frac{y_{i}+y_{j}}{2}\right]\right)^{-2}\right]-\left(\sinh \left[y_{i}\right]\right)^{-2} .
\end{aligned}
$$

We adopt $y_{n}=2 \alpha \tilde{r} n$ as an initial guess (as suggested in [17]) and stop the iteration process when either the sum of the magnitudes of the functions $F_{n}$ is less than some tolerance (typically $10^{-12}$ ) or the sum of the absolute values of the corrections $\delta y_{n}$ is less than some tolerance (typically $10^{-12}$ ). This method was found to always converge to the equilibrium.

The results are shown in Figure 3.1, where the imaginary parts of the poles of the coalescent steady states are plotted as functions of the parameter $\gamma$ introduced in section 2. Without loss of generality, here and in the rest of this section, only the case $r=1$ is presented. The case when $r$ is any other positive integer is very similar. For a coalescent pole solution, a pole can be uniquely identified by its imaginary part. We shall therefore use the notation $y_{n}^{k}$ for both the $n$th pole of the $k$-pole coalescent steady state and the imaginary part of this pole. We observe that

(i) The family of coalescent steady states consists of an infinitely countable number of solutions. We shall label each solution by the number of poles it possesses, namely, $u_{N}, N=0,1, \ldots, \infty$. We point out that the formulas (3.16)-(3.21) hold for coalescent steady states as well, except that now there shall be no $t$ dependence, with the only exception of $\varphi_{0}$ in (3.17), and that the subscript $N$ shall be added to the symbols $u$ and $\varphi$.

(ii) The coalescent steady state, $u_{N}$, exists on the semi-infinite interval $\gamma_{N}<$ $\gamma<\infty$. Here $\gamma_{N}=2(2 N-1)$ for $N>0$ and $\gamma_{0}=0$. Thus, the one-pole solution $u_{1}$ exists for $\gamma>2$; the two-pole solution $u_{2}$ exists for $\gamma>6$; the three-pole solution $u_{3}$ exists for $\gamma>10$; etc.

In physical space, the zero-pole coalescent steady state is the flat front. An $N$-pole coalescent steady state with $N>0$ is a front of a cusp-like shape that propagates with a constant speed. The depth of the "cusp" increases with increasing $\gamma$. A measure of the depth of the "cusp" of $\varphi_{N}$, or its amplitude, may be given by

$$
\Delta \varphi_{N}(\gamma) \equiv \max \varphi_{N}(\gamma)-\min \varphi_{N}(\gamma)=4 \alpha \sum_{n=1}^{N} \ln \left(\operatorname{coth} \frac{y_{n}}{2}\right),
$$

where use has been made of (3.17) to obtain the equality on the right-hand side. The dependence of $\Delta \varphi_{N}$ on $\gamma$ is shown in Figure 3.2. We note that at $\gamma=\gamma_{N}(N>0)$ the curve corresponding to the $N$-pole solution branches out from the curve corresponding to the $(N-1)$-pole solution. We thus have a cascade of infinitely many bifurcating solutions. The main goal of this work is to identify the stable and unstable branches of this bifurcation diagram. 


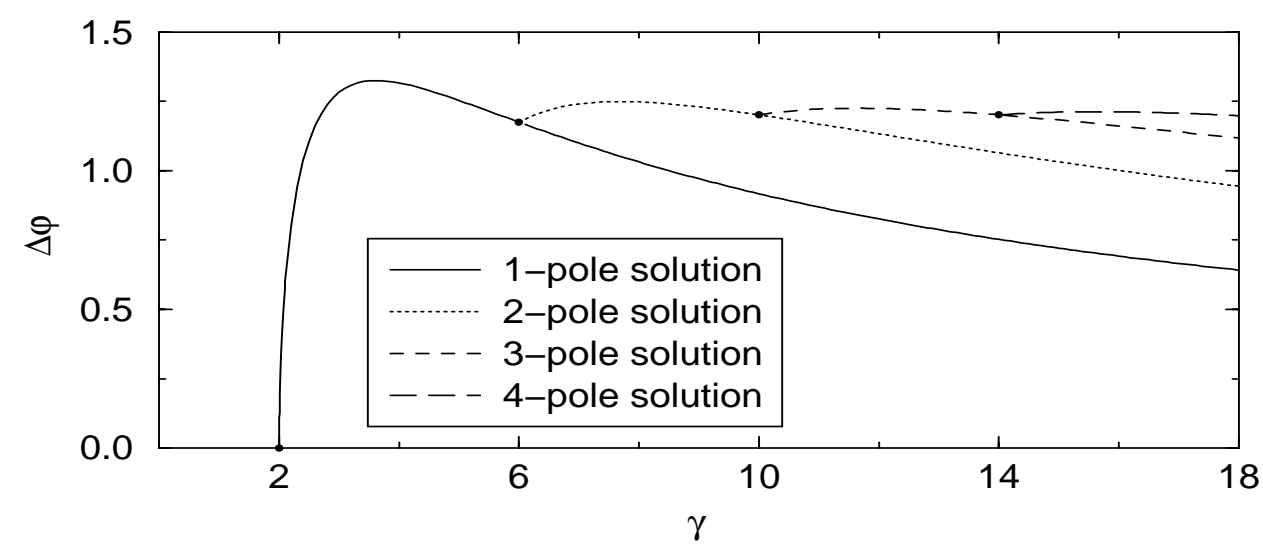

FIG. 3.2. The amplitudes, $\Delta \varphi$, of the $N$-pole coalescent steady states, with $N=0, \ldots, 4$ as functions of $\gamma$. The bifurcation points are marked with dots. The zero-pole solution coincides with the horizontal axis.

4. Eigenvalues and eigenfunctions. To examine the linear stability of coalescent steady states, the $N$-pole solution $u_{N}(x)$, or $\varphi_{N}(x, t)$, is perturbed, by writing

$$
u(x, t)=u_{N}(x)+v(x, t), \quad \text { or } \quad \varphi(x, t)=\varphi_{N}(x, t)+\psi(x, t),
$$

with $\|v\| \ll\left\|u_{N}\right\|,\|\psi\| \ll\left\|\varphi_{N}\right\|$. Here $\|\cdot\|$ denotes some appropriately chosen norm. Clearly, $v=\psi_{x}$.

4.1. Linearized equations. Substituting $u(x, t)$, or $\varphi(x, t)$, from (4.1) into (2.5), or (2.1), and then linearizing the result about the steady state $u_{N}(x)$ or $\varphi_{N}(x, t)$, yields

$$
v_{t}=\frac{1}{2} I\{v ; x\}+\alpha v_{x x}+\left(u_{N} v\right)_{x}
$$

or

$$
\psi_{t}=\frac{1}{2} I\{\psi ; x\}+\alpha \psi_{x x}+\left(\varphi_{N}\right)_{x} \psi_{x} .
$$

In this section, we deal only with the slope variable, $v$; the results for the displacement variable, $\psi$, will be written in subsection 4.3 below. In Fourier space, with

$$
v(x, t)=\sum_{\substack{k=-\infty \\ k \neq 0}}^{\infty} \hat{v}_{k}(t) e^{i \tilde{k} x}
$$

(4.2) takes the form

$$
\left(\hat{v}_{k}\right)_{t}=\frac{1}{2}|\tilde{k}| \hat{v}_{k}-\alpha \tilde{k}^{2} \hat{v}_{k}+\left[\widehat{\left(u_{N} v\right)_{x}}\right]_{k}, \quad k=-\infty, \ldots,-1,1, \ldots, \infty .
$$

Substituting (3.9), (4.4), and (3.21) into (4.5) yields an infinite system of equations

$$
\left(\hat{V}_{k}\right)_{t}=\frac{1}{2} \tilde{k} \sum_{\substack{m=-\infty, m \neq 0}}^{\infty} B_{k m} \hat{V}_{m}, \quad k=-\infty, \ldots,-1,1, \ldots, \infty
$$


where

$$
\hat{V}_{k}=e^{i k x_{c}} \hat{v}_{k}, \quad k=-\infty, \ldots,-1,1, \ldots, \infty
$$

can be thought of as the Fourier transform of $v\left(\tilde{x}+x_{c} / r\right)$ and $B_{k m}$ is an infinite matrix with entries given by

$$
\begin{aligned}
B_{k m} & =\operatorname{sgn}(k-m) \hat{u}_{N,|k-m|}^{\sin }, \quad k \neq m, \\
B_{k k} & =\operatorname{sgn}(k)-2 \alpha \tilde{k} .
\end{aligned}
$$

We point out that since all the entries of the matrix $B_{k m}$ are real, it suffices to consider only real solutions $\left\{\hat{V}_{k}\right\}$.

The linear system (4.6) can be decomposed into two infinite systems of linear equations. To show this, we introduce two sets of new variables: the symmetric variables

$$
\check{V}_{k}^{s}=\hat{V}_{k}-\hat{V}_{-k}, \quad k=1, \ldots, \infty,
$$

and the antisymmetric variables

$$
\check{V}_{k}^{a}=\hat{V}_{k}+\hat{V}_{-k}, \quad k=1, \ldots, \infty .
$$

(The label symmetric/antisymmetric will become clear later, in subsection 4.3.) The original variables can be uniquely expressed through $\check{V}_{k}^{s}$ and $\check{V}_{k}^{a}$ :

$$
\hat{V}_{k}= \begin{cases}\frac{1}{2}\left(\check{V}_{k}^{a}+\check{V}_{k}^{s}\right), & k=1, \ldots, \infty, \\ \frac{1}{2}\left(\check{V}_{-k}^{a}-\check{V}_{-k}^{s}\right), & k=-1, \ldots,-\infty .\end{cases}
$$

We subtract the $(-k)$ th equation of the linear system (4.6) from the $k$ th equation, insert (4.12) into the resulting expression, and obtain, after some manipulations, a system for symmetric variables $\check{V}_{k}^{s}$ only:

$$
\left(\check{V}_{k}^{s}\right)_{t}=\frac{1}{2} \tilde{k} \sum_{m=1}^{\infty} S_{k m} \check{V}_{m}^{s}, \quad k=1, \ldots, \infty .
$$

Here $S_{k m}$ is the infinite matrix with entries given by

$$
\begin{aligned}
S_{k m} & =\operatorname{sgn}(k-m) \hat{u}_{N,|k-m|}^{\sin }-\hat{u}_{N, k+m}^{\sin }, \quad k \neq m, \\
S_{k k} & =1-2 \alpha \tilde{k}-\hat{u}_{N, 2 k}^{\sin } .
\end{aligned}
$$

Similarly, we add the $k$ th and the $(-k)$ th equations of the linear system (4.6), substitute (4.12) into the resulting expression, and obtain, after some manipulations, a system for antisymmetric variables $\check{V}_{k}^{a}$ only:

$$
\left(\check{V}_{k}^{a}\right)_{t}=\frac{1}{2} \tilde{k} \sum_{m=1}^{\infty} A_{k m} \check{V}_{m}^{a}, \quad k=1, \ldots, \infty .
$$

Here $A_{k m}$ is the infinite matrix with entries given by

$$
\begin{aligned}
A_{k m} & =\operatorname{sgn}(k-m) \hat{u}_{N,|k-m|}^{\mathrm{sin}}+\hat{u}_{N, k+m}^{\sin }, \quad k \neq m, \\
A_{k k} & =1-2 \alpha \tilde{k}+\hat{u}_{N, 2 k}^{\mathrm{sin}} .
\end{aligned}
$$

We shall refer to the linear system (4.13) as the symmetric system and to the linear system (4.16) as the antisymmetric system. 
4.2. The eigenvalue problem. Our objective is to find the eigenvalues and eigenfunctions of the linear system (4.6). We therefore seek solutions of (4.6) in the form $\sim e^{\tilde{\lambda} t}$. In order to avoid additional notations, we shall denote the eigenfunctions with the same notation as the solution itself. Namely, $\hat{v}_{k}(t)=e^{\tilde{\lambda} t} \hat{v}_{k}$. Since, in the remainder of the paper, we will be interested only in the eigenfunctions, this convention will cause no confusion.

It is easy to see that if $\tilde{\lambda}$ is an eigenvalue of the full system (4.6) with the corresponding eigenfunction $\left\{\hat{V}_{k}\right\}$, then

(i) $\tilde{\lambda}$ is an eigenvalue of the symmetric system (4.13) with the eigenfunction $\left\{\check{V}_{k}^{s}\right\}$ defined by (4.10), provided $\hat{V}_{k} \neq \hat{V}_{-k}$ for some positive integral $k$, and

(ii) $\tilde{\lambda}$ is an eigenvalue of the antisymmetric system (4.16) with the eigenfunction $\left\{\check{V}_{k}^{a}\right\}$ defined by (4.11), provided $\hat{V}_{k} \neq-\hat{V}_{-k}$ for some positive integral $k$.

Conversely,

(i) if $\tilde{\lambda}$ is an eigenvalue of the symmetric system (4.13) with the eigenfunction $\left\{\check{V}_{k}^{s}\right\}$, then $\tilde{\lambda}$ is also an eigenvalue of the full system (4.6) with the eigenfunction

$$
\hat{V}_{k}= \begin{cases}\frac{1}{2} \check{V}_{k}^{s}, & k=1, \ldots, \infty \\ -\frac{1}{2} \check{V}_{-k}^{s}, & k=-1, \ldots,-\infty\end{cases}
$$

(ii) if $\tilde{\lambda}$ is an eigenvalue of the antisymmetric system (4.16) with the eigenfunction $\left\{\check{V}_{k}^{a}\right\}$, then $\tilde{\lambda}$ is also an eigenvalue of the full system (4.6) with the eigenfunction

$$
\hat{V}_{k}= \begin{cases}\frac{1}{2} \check{V}_{k}^{a}, & k=1, \ldots, \infty \\ \frac{1}{2} \check{V}_{-k}^{a}, & k=-1, \ldots,-\infty\end{cases}
$$

It therefore suffices to solve the eigenvalue problems for the symmetric (4.13) and the antisymmetric (4.16) systems. The two systems can be expressed in a unified form:

$$
2 \lambda \check{V}_{k}=k\left[(1-2 \tilde{\alpha} k) \check{V}_{k}+\sum_{m=1}^{k-1} \hat{u}_{N, k-m}^{\mathrm{sin}} \check{V}_{m}-\sum_{m=k+1}^{\infty} \hat{u}_{N, m-k}^{\mathrm{sin}} \check{V}_{m} \pm \sum_{m=1}^{\infty} \hat{u}_{N, m+k}^{\mathrm{sin}} \check{V}_{m}\right] ;
$$

where, here and hereafter, the upper/lower sign corresponds to the antisymmetric/symmetric system, respectively. It is convenient for the discussion below to use $\lambda=\tilde{\lambda} L / \pi$, consistent with our notation convention, noting that $\tilde{\lambda}$ is the eigenvalue of the linear system.

From now on we assume $r=1$; we shall comment on $r>1$ in Part II [15]. In examining (4.21) we found that there are two types of eigenvalues/eigenfunctions that we discuss in turn.

4.2.1. Type I. We seek eigenfunctions of the form

$$
\check{V}_{k}=k \sum_{n=1}^{N} A_{n} e^{-k y_{n}}, \quad k=1, \ldots, \infty
$$


with coefficients $A_{n}$ that remain to be determined. Substituting (3.20) and (4.22) into (4.21) one finds, after some lengthy algebra outlined in Appendix A, the system

$$
\begin{aligned}
\sum_{n=1}^{N} e^{-k y_{n}}\left\{\lambda A_{n}-\tilde{\alpha}\left[\sum_{\substack{l=1 \\
l \neq n}}^{N}\left(A_{l}-A_{n}\right) \frac{1}{2 \sinh ^{2}\left(\frac{y_{l}-y_{n}}{2}\right)}\right.\right. \\
\left.\left.\quad \pm \sum_{l=1}^{N}\left(A_{l} \mp A_{n}\right) \frac{1}{2 \sinh ^{2}\left(\frac{y_{l}+y_{n}}{2}\right)}\right]\right\}=0 .
\end{aligned}
$$

Equation (4.23) must hold for all $k=1, \ldots, \infty$. Since the expression in the curly brackets does not depend on $k$, we conclude that the infinite linear system (4.23) is satisfied if and only if this expression vanishes. The infinite dimensional linear system (4.23) has thus been reduced to the $N$-dimensional linear system

$$
\begin{array}{r}
\lambda A_{n}=\tilde{\alpha}\left[\sum_{\substack{l=1 \\
l \neq n}}^{N}\left(A_{l}-A_{n}\right) \frac{1}{2 \sinh ^{2}\left(\frac{y_{l}-y_{n}}{2}\right)} \pm \sum_{l=1}^{N}\left(A_{l} \mp A_{n}\right) \frac{1}{2 \sinh ^{2}\left(\frac{y_{l}+y_{n}}{2}\right)}\right], \\
n=1, \ldots, N .
\end{array}
$$

For the antisymmetric system it simplifies to

$$
\lambda A_{n}=\tilde{\alpha} \sum_{\substack{l=1 \\ l \neq n}}^{N} \frac{1}{2}\left[\frac{1}{\sinh ^{2}\left(\frac{y_{l}-y_{n}}{2}\right)}+\frac{1}{\sinh ^{2}\left(\frac{y_{l}+y_{n}}{2}\right)}\right]\left(A_{l}-A_{n}\right), \quad n=1, \ldots, N
$$

and for the symmetric system it simplifies to

$$
\lambda A_{n}=\tilde{\alpha} \sum_{l=1}^{N} \frac{\partial F_{n}}{\partial y_{l}} A_{l}, \quad n=1, \ldots, N,
$$

with $\frac{\partial F_{n}}{\partial y_{l}}$, the Jacobian matrix, presented in (3.29).

Next we show that the following properties hold for the eigenvalues of (4.25) and (4.26) for all values of $\tilde{\alpha}<\tilde{\alpha}_{N}$ :

(i) There are $N$ eigenvalues of the symmetric system and $N$ eigenvalues of the antisymmetric system.

(ii) All eigenvalues of either system are real.

(iii) All eigenvalues of the antisymmetric system are nonpositive and all eigenvalues of the symmetric system are negative. The antisymmetric system always possesses a zero eigenvalue associated with a trivial translational mode.

The first property is obvious. The second property immediately follows from the fact that the matrices of both systems, (4.25) and (4.26), are symmetric matrices.

To prove the third property we use Geršgorin's theorem (cf. [20, Theorem 6.1.1]) which states that every eigenvalue of an $n$-by- $n$ matrix $\left\{a_{i j}\right\}$ lies in at least one of the $n$ discs with centers at $a_{i i}$ and radii

$$
R_{i}=\sum_{\substack{j=1 \\ j \neq i}}^{n}\left|a_{i j}\right|, \quad i=1, \ldots, n
$$


in the complex plane. Then all the eigenvalues of $\left\{a_{i j}\right\}$ are located in the union of $n$ discs, $\bigcup_{i=1}^{n}\left\{z:\left|z-a_{i i}\right| \leq R_{i}\right\}$.

For the antisymmetric system (4.25), we have

$$
\begin{aligned}
& a_{i i}=-\tilde{\alpha} \sum_{\substack{j=1 \\
j \neq i}}^{N} \frac{1}{2}\left[\frac{1}{\sinh ^{2}\left(\frac{y_{j}-y_{i}}{2}\right)}+\frac{1}{\sinh ^{2}\left(\frac{y_{j}+y_{i}}{2}\right)}\right]<0, \\
& R_{i}=\tilde{\alpha} \sum_{\substack{j=1 \\
j \neq i}}^{N} \frac{1}{2}\left[\frac{1}{\sinh ^{2}\left(\frac{y_{j}-y_{i}}{2}\right)}+\frac{1}{\sinh ^{2}\left(\frac{y_{j}+y_{i}}{2}\right)}\right]=-a_{i i}
\end{aligned}
$$

for $i=1, \ldots, N$. Thus, every one of the $n$ discs lies in the left half-plane and touches the imaginary axis only at one point. We conclude that all the eigenvalues of the antisymmetric system are necessarily nonnegative. It is obvious, however, that the system (4.25) always (for all $N$ ) possesses a zero eigenvalue, $\lambda_{1}^{N, a}=0$, with the corresponding eigenfunction $\check{V}_{1, k}^{N, a}=k A \sum_{n=1}^{N} e^{-k y_{n}}$ (where $A$ is a constant). This eigenfunction is a translational mode which can be expressed in terms of the $N$-pole solution itself, namely, $v_{1}^{N, a}(x)=$ const $\frac{d}{d x} u_{N}(x)$. This eigenmode stems from the Galilean invariance of the evolution equation.

For the symmetric system (4.26), we have

$$
\begin{aligned}
a_{i i} & =\tilde{\alpha} \frac{\partial F_{i}}{\partial y_{i}} \\
& =-\tilde{\alpha}\left\{\frac{1}{2} \sum_{\substack{j=1 \\
j \neq i}}^{N}\left[\left(\sinh \left[\frac{y_{i}-y_{j}}{2}\right]\right)^{-2}+\left(\sinh \left[\frac{y_{i}+y_{j}}{2}\right]\right)^{-2}\right]+\left(\sinh \left[y_{i}\right]\right)^{-2}\right\}<0, \\
R_{i} & =\tilde{\alpha} \sum_{\substack{j=1 \\
j \neq i}}^{N} \frac{\partial F_{i}}{\partial y_{j}} \\
& =\tilde{\alpha} \sum_{\substack{j=1 \\
j \neq i}}^{N} \frac{1}{2}\left[\left(\sinh \left[\frac{y_{i}-y_{j}}{2}\right]\right)^{-2}-\left(\sinh \left[\frac{y_{i}+y_{j}}{2}\right]\right)^{-2}\right]<-a_{i i}
\end{aligned}
$$

for $i=1, \ldots, N$. Thus every one of the $n$ discs lies completely in the open left halfplane never touching the imaginary axis. We conclude that all the eigenvalues of the symmetric system (4.26) are negative.

By constructing the eigenvalues of the $N$-dimensional linear systems (4.25) and $(4.26)$, we found that two more properties hold for both systems for all values of $\tilde{\alpha}<\tilde{\alpha}_{N}$ :

(iv) All $N$ eigenvalues are mutually distinct.

(v) All eigenvalues of either system, with the exception of $\lambda_{1}^{N, a}$, are monotonically increasing functions of $\tilde{\alpha}$. 
The properties (i)-(iv) lead to the conclusion that the spectrum of the antisymmetric system (4.25) is

$$
0=\lambda_{1}^{N, a}>\lambda_{2}^{N, a}>\cdots>\lambda_{N}^{N, a}
$$

and the spectrum of the symmetric system (4.26) is

$$
0>\lambda_{1}^{N, s}>\lambda_{2}^{N, s}>\cdots>\lambda_{N}^{N, s} .
$$

We note that the order, as well as the strict inequalities, in (4.32) and (4.33) is always retained.

4.2.2. Type II. We seek eigenfunctions of the form

$$
\check{V}_{k}=\check{V}_{N+S, k}^{N}= \begin{cases}k \sum_{n=1}^{N} A_{n} e^{-k y_{n}} & \text { if } k>S, \\ k \sum_{n=1}^{N} A_{n} e^{-k y_{n}}+w_{k} & \text { if } k \leq S,\end{cases}
$$

where $S=1, \ldots, \infty$, with coefficients $A_{n}(n=1, \ldots, N)$ and $w_{k}(k=1, \ldots, S)$ that remain to be determined $\left(w_{S} \neq 0\right)$. Substituting (3.20) and (4.34) into (4.21) one finds, after some lengthy algebra outlined in Appendix B, the two systems

$$
\begin{array}{r}
\left.\frac{1}{2} k[1-(k+2 N) 2 \tilde{\alpha}]-\lambda\right\} w_{k}=4 \tilde{\alpha} k \sum_{m=k+1}^{S}\left(\sum_{n=1}^{N} \cosh \left[(m-k) y_{n}\right]\right) w_{m} \\
k=1, \ldots, S
\end{array}
$$

$$
\begin{aligned}
\sum_{n=1}^{N} e^{-k y_{n}}\left\{\lambda A_{n}\right. & -\tilde{\alpha}\left[\sum_{\substack{l=1 \\
l \neq n}}^{N}\left(A_{l}-A_{n}\right) \frac{1}{2 \sinh ^{2}\left(\frac{y_{l}-y_{n}}{2}\right)} \pm \sum_{l=1}^{N}\left(A_{l} \mp A_{n}\right) \frac{1}{2 \sinh ^{2}\left(\frac{y_{l}+y_{n}}{2}\right)}\right. \\
& \left.\left.+2 \sum_{m=1}^{S}\left(e^{m y_{n}} \pm e^{-m y_{n}}\right) w_{m}\right]\right\}=0, \quad k=S+1, \ldots, \infty .
\end{aligned}
$$

We start by examining the system of equations (4.35). The right-hand side of the last equation, which corresponds to $k=S$, is zero. This implies that the expression inside the curly brackets on the left-hand side vanishes (because $w_{S} \neq 0$ ). We have thus obtained an expression for the $(N+S)$ th eigenvalue, namely,

$$
\lambda_{N+S}^{N}=\frac{1}{2} S[1-(S+2 N) 2 \tilde{\alpha}], \quad S=1, \ldots, \infty .
$$

It should be pointed out that we start numbering the eigenvalues of type II with $N+1$; the first $N$ eigenvalues are the eigenvalues of type I (found in the preceding subsection). The remaining $S-1$ equations become

$$
\begin{array}{r}
\frac{1}{2}[k-S][1-(k+S+2 N) 2 \tilde{\alpha}] w_{k}=4 \tilde{\alpha} k \sum_{m=k+1}^{S}\left(\sum_{n=1}^{N} \cosh \left[(m-k) y_{n}\right]\right) w_{m}, \\
k=1, \ldots, S-1
\end{array}
$$


or, equivalently,

$$
\begin{array}{r}
{\left[\frac{k-S}{k+S}\right] \lambda_{N+S+k}^{N} w_{k}=4 \tilde{\alpha} k \sum_{m=k+1}^{S}\left(\sum_{n=1}^{N} \cosh \left[(m-k) y_{n}\right]\right) w_{m}} \\
k=1, \ldots, S-1 .
\end{array}
$$

These $S-1$ equations determine the $S-1$ unknowns, $w_{1}, \ldots, w_{S-1} ; w_{S}$ is an arbitrary constant. Because the coefficients on the left-hand side never vanish, except for the values of $\tilde{\alpha}$, where $\lambda_{N+S+k}^{N}=0$, the $S-1$ equations (4.39) can be solved recursively starting from the last $(k=S-1)$ equation and going down to the first $(k=1)$ equation. We thus obtain expressions for $w_{k}$ as linear combinations of the remaining $w_{m}$ :

$$
w_{m}=C W_{m}(\tilde{\alpha}, N, S), \quad m=1, \ldots, S-1
$$

for some function $W_{m}$ that depends on $\tilde{\alpha}, N$, and $S$, where $C=w_{S}$. Finally, we point out that $\lambda_{N+S}^{N}$ is an eigenvalue for both the symmetric and the antisymmetric systems, and that $w_{m}, m=1, \ldots, S-1$ given by (4.40) are the same for both systems.

We note that for $N=0$ there is no equation (4.36). In this case the righthand side of (4.35) vanishes, again giving (4.37) for the eigenvalues with $w_{m}=0$ for $m=1, \ldots, S-1$, while $w_{S}$ remains an arbitrary constant.

We now consider the system of (4.36) (relevant for $N \geq 1$ ), which must hold for all $k=S+1, \ldots, \infty$. Since the expression in the curly brackets does not depend on $k$, we conclude that the infinite linear system (4.36) is satisfied if and only if this expression vanishes. The infinite dimensional linear system (4.36) has thus been reduced to the $N$-dimensional linear system

$$
\begin{aligned}
\lambda A_{n}=\tilde{\alpha}\left[\sum_{\substack{l=1 \\
l \neq n}}^{N}\left(A_{l}-A_{n}\right)\right. & \frac{1}{2 \sinh ^{2}\left(\frac{y_{l}-y_{n}}{2}\right)} \pm \sum_{l=1}^{N}\left(A_{l} \mp A_{n}\right) \frac{1}{2 \sinh ^{2}\left(\frac{y_{l}+y_{n}}{2}\right)} \\
& \left.+2 \sum_{m=1}^{S}\left(e^{m y_{n}} \pm e^{-m y_{n}}\right) w_{m}\right], \quad n=1, \ldots, N .
\end{aligned}
$$

Substituting (4.37) for $\lambda$ and (4.40) for $w_{m}(m=1, \ldots, S-1)$ we obtain a system of $N$ nonhomogeneous linear equations

$$
\begin{array}{r}
\lambda_{N+S}^{N} A_{n}-\tilde{\alpha}\left[\sum_{\substack{l=1 \\
l \neq n}}^{N}\left(A_{l}-A_{n}\right) \frac{1}{2 \sinh ^{2}\left(\frac{y_{l}-y_{n}}{2}\right)} \pm \sum_{l=1}^{N}\left(A_{l} \mp A_{n}\right) \frac{1}{2 \sinh ^{2}\left(\frac{y_{l}+y_{n}}{2}\right)}\right] \\
=2 \tilde{\alpha} C \sum_{m=1}^{S}\left(e^{m y_{n}} \pm e^{-m y_{n}}\right) W_{m}(\tilde{\alpha}, N, S), \quad n=1, \ldots, N
\end{array}
$$

for the $N$ unknowns $A_{n}$, where $W_{S}(\tilde{\alpha}, N, S) \equiv 1$. The matrix of this linear system coincides with the matrix of the homogeneous linear system (4.24) discussed earlier, both being functions of $\lambda$. Because all the eigenvalues of type I are monotonically increasing functions of $\tilde{\alpha}$, whereas the eigenvalues of type II are monotonically decreasing functions of $\tilde{\alpha}$ (see (4.37)), the determinant of the system vanishes at most at $N$ values of $\tilde{\alpha}$, namely, where the eigenvalues of type I intersect the eigenvalues of 
type II. At all other values of $\tilde{\alpha}$ the system (4.42) has a unique solution: for a given eigenvalue $\lambda_{N+S}^{N}$, given by (4.37), there is one and only one eigenfunction for the symmetric system and one and only one eigenfunction for the antisymmetric system.

For completeness, we note that the system (4.42) simplifies as follows: for the antisymmetric system it takes the form

$$
\begin{aligned}
\lambda_{N+S}^{N} A_{n} & -\tilde{\alpha} \sum_{\substack{l=1 \\
l \neq n}}^{N} \frac{1}{2}\left[\frac{1}{\sinh ^{2}\left(\frac{y_{l}-y_{n}}{2}\right)}+\frac{1}{\sinh ^{2}\left(\frac{y_{l}+y_{n}}{2}\right)}\right]\left(A_{l}-A_{n}\right) \\
= & 4 \tilde{\alpha} C \sum_{m=1}^{S} \cosh \left(m y_{n}\right) W_{m}(\tilde{\alpha}, N, S), \quad n=1, \ldots, N,
\end{aligned}
$$

and for the symmetric system,

$$
\begin{array}{r}
\lambda_{N+S}^{N} A_{n}-\tilde{\alpha} \sum_{l=1}^{N} \frac{\partial F_{n}}{\partial y_{l}} A_{l}=4 \tilde{\alpha} C \sum_{m=1}^{S} \sinh \left(m y_{n}\right) W_{m}(\tilde{\alpha}, N, S), \\
n=1, \ldots, N,
\end{array}
$$

with $\frac{\partial F_{n}}{\partial y_{l}}$, the Jacobian matrix, presented in (3.29).

We end this subsection with a list of properties of the eigenvalues of type II which follow immediately from (4.37) and hold for all values of $\tilde{\alpha}<\tilde{\alpha}_{N}$. They are counterparts of the properties of eigenvalues of type I listed in the previous subsection.

(i) There is an infinitely countable number of the eigenvalues.

(ii) All eigenvalues are real.

(iii) All eigenvalues are negative for large values of $\tilde{\alpha}$ and positive for small values of $\tilde{\alpha}$. A typical eigenvalue, $\lambda_{N+S}^{N}$, changes its sign at $\tilde{\alpha}=\frac{1}{2(S+2 N)}$.

(iv) Any two eigenvalues, $\lambda_{N+S}^{N}$ and $\lambda_{N+M}^{N}$, assume the same value at one and only one point, $\tilde{\alpha}=\frac{1}{2(M+S+2 N)}$. They are mutually distinct for all other values of $\tilde{\alpha}$.

(v) All eigenvalues are linearly decreasing functions of $\tilde{\alpha}$.

4.3. Eigenfunctions in physical space. We summarize the results concerning eigenfunctions by providing their expressions in physical space. We start by noting that the decomposition (4.12) implies that the solution $v(x, t)$ can be expressed as $v(x, t)=v^{a}(x, t)+i v^{s}(x, t)$ with

$$
\begin{aligned}
& v^{s}(x, t)=\sum_{k=1}^{\infty} \check{V}_{k}^{s}(t) \sin \left[k\left(\tilde{x}-\frac{x_{c}}{r}\right)\right], \\
& v^{a}(x, t)=\sum_{k=1}^{\infty} \check{V}_{k}^{a}(t) \cos \left[k\left(\tilde{x}-\frac{x_{c}}{r}\right)\right] .
\end{aligned}
$$

This is obtained by simply inserting (4.12) and (4.7) into (4.4). The flame front perturbation can also be expressed in the form $\psi(x, t)=\psi^{a}(x, t)+i \psi^{s}(x, t)$ with

$$
\begin{gathered}
\psi^{s}(x, t)=\check{\Psi}_{0}^{s}(t)+\sum_{k=1}^{\infty} \check{\Psi}_{k}^{s}(t) \cos \left[k\left(\tilde{x}-\frac{x_{c}}{r}\right)\right], \quad \check{\Psi}_{k}^{s}=-\frac{\check{V}_{k}^{s}}{\tilde{k}}, \\
\psi^{a}(x, t)=\sum_{k=1}^{\infty} \check{\Psi}_{k}^{a}(t) \sin \left[k\left(\tilde{x}-\frac{x_{c}}{r}\right)\right], \quad \check{\Psi}_{k}^{a}=\frac{\check{V}_{k}^{a}}{\tilde{k}} .
\end{gathered}
$$


The notation here reflects the fact that the superscripts ${ }^{s}$ and ${ }^{a}$ represent the symmetric and antisymmetric, with respect to $\tilde{x}=x_{c} / r$, contributions to the perturbation of the flame front.

This decomposition leads to the following expressions for the eigenfunctions in physical space. For the eigenfunctions of type I, we insert (4.22) into (4.45) and (4.46) to obtain the symmetric and antisymmetric perturbations to the slope of the flame front,

$$
\begin{aligned}
& v^{N, s}(x)=\frac{1}{2} \sum_{n=1}^{N} A_{n} \frac{\sinh y_{n} \sin \left(\tilde{x}-x_{c}\right)}{\left(\cosh y_{n}-\cos \left(\tilde{x}-x_{c}\right)\right)^{2}}, \\
& v^{N, a}(x)=\frac{1}{2} \sum_{n=1}^{N} A_{n} \frac{\cosh y_{n} \cos \left(\tilde{x}-x_{c}\right)-1}{\left(\cosh y_{n}-\cos \left(\tilde{x}-x_{c}\right)\right)^{2}},
\end{aligned}
$$

and into (4.47) and (4.48) to obtain the symmetric and antisymmetric perturbations to the displacement of the flame front,

$$
\begin{gathered}
\tilde{\psi}^{N, s}(x)=-\frac{1}{2} \sum_{n=1}^{N} A_{n} \frac{\cos \left(\tilde{x}-x_{c}\right)-e^{-y_{n}}}{\cosh y_{n}-\cos \left(\tilde{x}-x_{c}\right)}, \\
\tilde{\psi}^{N, a}(x)=\frac{1}{2} \sum_{n=1}^{N} A_{n} \frac{\sin \left(\tilde{x}-x_{c}\right)}{\cosh y_{n}-\cos \left(\tilde{x}-x_{c}\right)} .
\end{gathered}
$$

For the eigenfunctions of type II, we insert (4.34) into (4.45) and (4.46) to obtain the symmetric and antisymmetric perturbations to the slope of the flame front,

$$
\begin{aligned}
& \text { (4.53) } v_{N+S}^{N, s}(x)=\frac{1}{2} \sum_{n=1}^{N} A_{n} \frac{\sinh y_{n} \sin \left(\tilde{x}-x_{c}\right)}{\left(\cosh y_{n}-\cos \left(\tilde{x}-x_{c}\right)\right)^{2}}+\sum_{k=1}^{S} w_{k} \sin \left[k\left(\tilde{x}-x_{c}\right)\right], \\
& \text { (4.54) } v_{N+S}^{N, a}(x)=\frac{1}{2} \sum_{n=1}^{N} A_{n} \frac{\cosh y_{n} \cos \left(\tilde{x}-x_{c}\right)-1}{\left(\cosh y_{n}-\cos \left(\tilde{x}-x_{c}\right)\right)^{2}}+\sum_{k=1}^{S} w_{k} \cos \left[k\left(\tilde{x}-x_{c}\right)\right],
\end{aligned}
$$

and into (4.47) and (4.48) to obtain the symmetric and antisymmetric perturbations to the displacement of the flame front,

$$
\begin{aligned}
& \tilde{\psi}_{N+S}^{N, s}(x)=-\frac{1}{2} \sum_{n=1}^{N} A_{n} \frac{\cos \left(\tilde{x}-x_{c}\right)-e^{-y_{n}}}{\cosh y_{n}-\cos \left(\tilde{x}-x_{c}\right)}-\sum_{k=1}^{S} \frac{w_{k}}{k} \cos \left[k\left(\tilde{x}-x_{c}\right)\right] \\
& \tilde{\psi}_{N+S}^{N, a}(x)=\frac{1}{2} \sum_{n=1}^{N} A_{n} \frac{\sin \left(\tilde{x}-x_{c}\right)}{\cosh y_{n}-\cos \left(\tilde{x}-x_{c}\right)}+\sum_{k=1}^{S} \frac{w_{k}}{k} \sin \left[k\left(\tilde{x}-x_{c}\right)\right]
\end{aligned}
$$


5. Conclusions. The spectrum of the full linear system (4.6), associated with an $N$-pole coalescent steady state $u_{N}(x)$, consists of two sets of eigenvalues of type I, the symmetric set and the antisymmetric set, and one set of eigenvalues of type II.

Either set of eigenvalues of type I consists of $N$ mutually distinct eigenvalues, ordered as in (4.32) or (4.33). All eigenvalues of the symmetric set are distinct from the eigenvalues of the antisymmetric set, for all $\gamma>\gamma_{N}$. Each eigenvalue of type I is a simple eigenvalue; there is only one eigenfunction associated with it. The eigenfunction associated with an eigenvalue of the symmetric set is symmetric. It is of the form (4.22) in Fourier space (or (4.49) for the slope and (4.51) for the displacement in physical space) with $A_{n}$ being solutions of the linear system (4.26). The eigenfunction associated with an eigenvalue of the antisymmetric set is antisymmetric. It is of the form (4.22) in Fourier space (or (4.50) for the slope and (4.52) for the displacement in physical space) with $A_{n}$ solutions of the linear system (4.25).

There are infinitely many eigenvalues of type II, given by (4.37). Each eigenvalue of type II has multiplicity two; there are two different eigenfunctions associated with it - one symmetric and one antisymmetric. The symmetric eigenfunction is of the form (4.34) in Fourier space (or (4.53) for the slope and (4.55) for the displacement in physical space) with $A_{n}$ solutions of the linear system (4.44) and $w_{k}$ given by (4.40). The antisymmetric eigenfunction is of the form (4.34) in Fourier space (or (4.54) for the slope and (4.56) for the displacement in physical space) with $A_{n}$ solutions of the linear system (4.43) and $w_{k}$ given by (4.40). To substantiate this, we solved the eigenvalue problem for the linear system (4.6) numerically for various values of $N$ and $\tilde{\alpha}$. The matrix was first truncated at some cutoff $k=k^{c}$. The resulting $\left(k^{c} \times k^{c}\right)$ dimensional matrix was then solved for the eigenvalues using standard computational linear algebra techniques (cf. [21]). By increasing $k^{c}$ we ensured the convergence of the low-order eigenvalues. For all values of $N$ and $\tilde{\alpha}$ that we tested, we found exact (within numerical error) agreement between the numerical and our analytical results.

In Part II [15] we examine the properties of the eigenvalues and eigenfunctions as they relate to the stability of an $N$-pole coalescent steady state. Being based on analytical expressions, our results resolve unequivocally the earlier controversies reported in the literature and discussed in section 1 . We also examine the dependence of the eigenvalues and eigenfunctions on the parameter $\gamma$, which provides insight on the behavior of the nonlinear evolution equation (2.1) and, consequently, on the dynamics of a hydrodynamically unstable planar flame.

Appendix A. In this appendix we show the main steps in the derivation of (4.23). Substituting (3.20) and (4.22) into (4.21) we obtain

(A.1)

$$
\begin{aligned}
2 \lambda k \sum_{n=1}^{N} A_{n} e^{-k y_{n}}=k\left\{(1-2 \alpha k) k \sum_{n=1}^{N} A_{n} e^{-k y_{n}}\right. \\
+4 \alpha\left[\sum_{m=1}^{k-1} \sum_{l=1}^{N} e^{-k y_{l}} e^{m y_{l}} m \sum_{n=1}^{N} A_{n} e^{-m y_{n}}\right. \\
\left.\left.\quad-\sum_{m=k+1}^{\infty} \sum_{l=1}^{N} e^{k y_{l}} e^{-m y_{l}} m \sum_{n=1}^{N} A_{n} e^{-m y_{n}} \pm \sum_{m=1}^{\infty} \sum_{l=1}^{N} e^{-k y_{l}} e^{-m y_{l}} m \sum_{n=1}^{N} A_{n} e^{-m y_{n}}\right]\right\} .
\end{aligned}
$$

We denote the three terms in the square brackets by $S_{1}, S_{2}$, and $S_{3}$, respectively.

Interchanging the order of summation in $S_{1}$ and then splitting the inner sum, one 
obtains

$$
\begin{aligned}
S_{1} & =\sum_{l=1}^{N} e^{-k y_{l}} \sum_{n=1}^{N} A_{n} \sum_{m=1}^{k-1} m e^{-m\left(y_{n}-y_{l}\right)} \\
& =\sum_{l=1}^{N} e^{-k y_{l}}\left\{\sum_{\substack{n=1 \\
n \neq l}}^{N} A_{n} \sum_{m=1}^{k-1} m e^{-m\left(y_{n}-y_{l}\right)}+A_{l} \sum_{m=1}^{k-1} m\right\} .
\end{aligned}
$$

Next, we sum up with respect to $m$ using the following two relations:

$$
\sum_{m=1}^{k-1} m=\frac{k(k-1)}{2},
$$

$$
\begin{aligned}
\sum_{m=1}^{k-1} m e^{-m\left(y_{n}-y_{l}\right)} & =\frac{1}{4 \sinh ^{2}\left(\frac{y_{n}-y_{l}}{2}\right)} \\
& -e^{-\left(y_{n}-y_{l}\right) k}\left\{k \frac{1}{2}\left[\operatorname{coth}\left(\frac{y_{n}-y_{l}}{2}\right)+1\right]+\frac{1}{4 \sinh ^{2}\left(\frac{y_{n}-y_{l}}{2}\right)}\right\}
\end{aligned}
$$

where the latter is obtained by differentiating the geometric progression formula

$$
\begin{aligned}
\sum_{m=1}^{k-1} m e^{-m y} & =-\frac{d}{d y} \sum_{m=1}^{k-1} e^{-m y}=-\frac{d}{d y}\left[\frac{e^{-y}-e^{-y k}}{1-e^{-y}}\right] \\
& =\frac{e^{-y}}{\left(1-e^{-y}\right)^{2}}-e^{-y k}\left\{k \frac{1}{\left(1-e^{-y}\right)}+\frac{e^{-y}}{\left(1-e^{-y}\right)^{2}}\right\} \\
& =\frac{1}{4 \sinh ^{2}\left(\frac{y}{2}\right)}-e^{-y k}\left\{k \frac{1}{2}\left[\operatorname{coth}\left(\frac{y}{2}\right)+1\right]+\frac{1}{4 \sinh ^{2}\left(\frac{y}{2}\right)}\right\} .
\end{aligned}
$$

After rearranging terms one obtains

$$
\begin{aligned}
S_{1} & =\sum_{l=1}^{N} e^{-k y_{l}}\left\{\sum_{\substack{n=1 \\
n \neq l}}^{N} A_{n} \frac{1}{4 \sinh ^{2}\left(\frac{y_{n}-y_{l}}{2}\right)}+A_{l} \frac{k(k-1)}{2}\right\} \\
& -\sum_{n=1}^{N} A_{n} e^{-k y_{n}} \sum_{\substack{l=1 \\
l \neq n}}^{N}\left\{k \frac{1}{2}\left[\operatorname{coth}\left(\frac{y_{n}-y_{l}}{2}\right)+1\right]+\frac{1}{4 \sinh ^{2}\left(\frac{y_{n}-y_{l}}{2}\right)}\right\} .
\end{aligned}
$$

Finally, renaming the indices $l$ as $n$ and $n$ as $l$ in the first term of (A.6) leads to the expression

$$
\begin{array}{r}
S_{1}=\sum_{n=1}^{N} e^{-k y_{n}}\left\{A_{n} \frac{k(k-1)}{2}-A_{n} k \sum_{\substack{l=1 \\
l \neq n}}^{N} \frac{1}{2}\left[\operatorname{coth}\left(\frac{y_{n}-y_{l}}{2}\right)+1\right]\right. \\
\left.+\sum_{\substack{l=1 \\
l \neq n}}^{N}\left(A_{l}-A_{n}\right) \frac{1}{4 \sinh ^{2}\left(\frac{y_{l}-y_{n}}{2}\right)}\right\} .
\end{array}
$$


In $S_{2}$ we change the order of summation and then sum up with respect to $m$ to obtain

$$
\begin{aligned}
S_{2} & =\sum_{n=1}^{N} A_{n} \sum_{l=1}^{N} e^{k y_{l}} \sum_{m=k+1}^{\infty} m e^{-m\left(y_{n}+y_{l}\right)} \\
& =\sum_{n=1}^{N} A_{n} \sum_{l=1}^{N} e^{k y_{l}} e^{-\left(y_{n}+y_{l}\right) k}\left\{\frac{1}{4 \sinh ^{2}\left(\frac{y_{n}+y_{l}}{2}\right)}+k \frac{1}{2}\left[\operatorname{coth}\left(\frac{y_{n}+y_{l}}{2}\right)-1\right]\right\} \\
& =\sum_{n=1}^{N} e^{-k y_{n}} A_{n} \sum_{l=1}^{N}\left\{\frac{1}{4 \sinh ^{2}\left(\frac{y_{l}+y_{n}}{2}\right)}+k \frac{1}{2}\left[\operatorname{coth}\left(\frac{y_{n}+y_{l}}{2}\right)-1\right]\right\},
\end{aligned}
$$

where a sequence of steps analogous to those used in obtaining (A.5) has been performed; in particular we used the relation

$$
\begin{aligned}
\sum_{m=k+1}^{\infty} m e^{-m y} & =-\frac{d}{d y} \sum_{m=k+1}^{\infty} e^{-m y}=-\frac{d}{d y}\left[\frac{e^{-y(k+1)}}{1-e^{-y}}\right] \\
& =e^{-y k}\left\{\frac{e^{-y}}{\left(1-e^{-y}\right)^{2}}+k \frac{e^{-y}}{1-e^{-y}}\right\} \\
& =e^{-y k}\left\{\frac{1}{4 \sinh ^{2}\left(\frac{y}{2}\right)}+k \frac{1}{2}\left[\operatorname{coth}\left(\frac{y}{2}\right)-1\right]\right\} .
\end{aligned}
$$

In $S_{3}$ we change the order of summation and then sum up with respect to $m$ to obtain

$$
S_{3}=\sum_{l=1}^{N} e^{-k y_{l}} \sum_{n=1}^{N} A_{n} \sum_{m=1}^{\infty} m e^{-m\left(y_{n}+y_{l}\right)}=\sum_{l=1}^{N} e^{-k y_{l}} \sum_{n=1}^{N} A_{n} \frac{1}{4 \sinh ^{2}\left(\frac{y_{l}+y_{n}}{2}\right)}
$$

where (A.9) with $k=0$ has been used to sum up with respect to $m$.

Now, we combine the expressions (A.7) and (A.8) and regroup the terms to obtain

$$
\begin{aligned}
S_{1}-S_{2} & =\sum_{n=1}^{N} e^{-k y_{n}}\left(A _ { n } \left\{\frac{k^{2}}{2}-\frac{k}{2}\left[\sum_{\substack{l=1 \\
l \neq n}}^{N}\left(\operatorname{coth}\left(\frac{y_{n}-y_{l}}{2}\right)+\operatorname{coth}\left(\frac{y_{n}+y_{l}}{2}\right)\right)\right.\right.\right. \\
& \left.\left.\left.+\operatorname{coth}\left(y_{n}\right)\right]\right\}+\sum_{\substack{l=1 \\
l \neq n}}^{N}\left(A_{l}-A_{n}\right) \frac{1}{4 \sinh ^{2}\left(\frac{y_{l}-y_{n}}{2}\right)}-A_{n} \sum_{l=1}^{N} \frac{1}{4 \sinh ^{2}\left(\frac{y_{l}+y_{n}}{2}\right)}\right)
\end{aligned}
$$

which can be further simplified, using (3.22) and (3.15), to

$$
\begin{aligned}
S_{1}-S_{2} & =\sum_{n=1}^{N} e^{-k y_{n}}\left(-A_{n} \frac{k}{4 \alpha}(1-2 \alpha k)\right. \\
& \left.+\sum_{\substack{l=1 \\
l \neq n}}^{N}\left(A_{l}-A_{n}\right) \frac{1}{4 \sinh ^{2}\left(\frac{y_{l}-y_{n}}{2}\right)}-A_{n} \sum_{l=1}^{N} \frac{1}{4 \sinh ^{2}\left(\frac{y_{l}+y_{n}}{2}\right)}\right) .
\end{aligned}
$$


Finally, inserting (A.10) and (A.12) into (A.1) we arrive at (4.23).

Appendix B. In this appendix we show the main steps in the derivation of (4.35) and (4.36). Substituting (3.20) and (4.34) into (4.21) we obtain

$$
\begin{aligned}
2 \lambda k & \sum_{n=1}^{N} A_{n} e^{-k y_{n}}+2 \lambda w_{k}=k\left\{(1-2 \alpha k) k \sum_{n=1}^{N} A_{n} e^{-k y_{n}}\right. \\
& +4 \alpha\left[\sum_{m=1}^{k-1} \sum_{l=1}^{N} e^{-k y_{l}} e^{m y_{l}} m \sum_{n=1}^{N} A_{n} e^{-m y_{n}}-\sum_{m=k+1}^{\infty} \sum_{l=1}^{N} e^{k y_{l}} e^{-m y_{l}} m \sum_{n=1}^{N} A_{n} e^{-m y_{n}}\right. \\
& \left. \pm \sum_{m=1}^{\infty} \sum_{l=1}^{N} e^{-k y_{l}} e^{-m y_{l}} m \sum_{n=1}^{N} A_{n} e^{-m y_{n}}\right]+(1-2 \alpha k) w_{k} \\
& +4 \alpha\left[\sum_{m=1}^{k-1} \sum_{n=1}^{N} e^{-k y_{n}} e^{m y_{n}} w_{m}-\sum_{m=k+1}^{S} \sum_{n=1}^{N} e^{k y_{n}} e^{-m y_{n}} w_{m}\right. \\
& \left.\left. \pm \sum_{m=1}^{S} \sum_{n=1}^{N} e^{-k y_{n}} e^{-m y_{n}} w_{m}\right]\right\}, \quad k=1, \ldots, S,
\end{aligned}
$$

$$
\begin{aligned}
2 \lambda k & \sum_{n=1}^{N} A_{n} e^{-k y_{n}}=k\left\{(1-2 \alpha k) k \sum_{n=1}^{N} A_{n} e^{-k y_{n}}\right. \\
& +4 \alpha\left[\sum_{m=1}^{k-1} \sum_{l=1}^{N} e^{-k y_{l}} e^{m y_{l}} m \sum_{n=1}^{N} A_{n} e^{-m y_{n}}-\sum_{m=k+1}^{\infty} \sum_{l=1}^{N} e^{k y_{l}} e^{-m y_{l}} m \sum_{n=1}^{N} A_{n} e^{-m y_{n}}\right. \\
& \left. \pm \sum_{m=1}^{\infty} \sum_{l=1}^{N} e^{-k y_{l}} e^{-m y_{l}} m \sum_{n=1}^{N} A_{n} e^{-m y_{n}}\right] \\
& \left.+4 \alpha \sum_{n=1}^{N} e^{-k y_{n}}\left[\sum_{m=1}^{S}\left(e^{m y_{n}} \pm e^{-m y_{n}}\right) w_{m}\right]\right\}, \quad k=S+1, \ldots, \infty
\end{aligned}
$$

Equation (B.2) can be easily transformed to expression (4.36) following the steps outlined in Appendix A because, with the exception of one extra term, (B.2) is exactly (A.1).

The first two terms inside the curly brackets of (B.1) coincide with the expression inside the curly brackets in (A.1). We therefore simplify the former by following the steps of Appendix A. Next we change the order of summation in each of the three terms inside the second brackets of (B.1) and rewrite the first term inside the second brackets as follows:

$$
\sum_{n=1}^{N} e^{-k y_{n}} \sum_{m=1}^{k-1} e^{m y_{n}} w_{m}=\sum_{n=1}^{N} e^{-k y_{n}}\left(\sum_{m=1}^{S} e^{m y_{n}} w_{m}-\sum_{m=k+1}^{S} e^{m y_{n}} w_{m}\right)-N w_{k}
$$


After some regrouping, (B.1) becomes

$$
\begin{aligned}
2 \lambda w_{k} & +2 k \sum_{n=1}^{N} e^{-k y_{n}}\left\{\lambda A_{n}-\alpha\left[\sum_{\substack{l=1 \\
l \neq n}}^{N}\left(A_{l}-A_{n}\right) \frac{1}{2 \sinh ^{2}\left(\frac{y_{l}-y_{n}}{2}\right)}\right.\right. \\
& \left.\left. \pm \sum_{l=1}^{N}\left(A_{l} \mp A_{n}\right) \frac{1}{2 \sinh ^{2}\left(\frac{y_{l}+y_{n}}{2}\right)}+2 \sum_{m=1}^{S}\left(e^{m y_{n}} \pm e^{-m y_{n}}\right) w_{m}\right]\right\} \\
& =k\left\{(1-2 \alpha k-4 \alpha N) w_{k}\right. \\
& \left.-4 \alpha \sum_{n=1}^{N}\left[e^{-k y_{n}} \sum_{m=k+1}^{S} e^{m y_{n}} w_{m}+e^{k y_{n}} \sum_{m=k+1}^{S} e^{-m y_{n}} w_{m}\right]\right\} .
\end{aligned}
$$

Due to (4.36) only the first term survives in the left-hand side of (B.4). Finally, changing the order of summation in the second term inside the curly brackets on the right-hand side of (B.4) we arrive at the expression (4.35).

\section{REFERENCES}

[1] G. I. Sivashinsky, Nonlinear analysis of hydrodynamic instability in laminar flames-I. Derivation of basic equations, Acta Astronautica, 4 (1977), pp. 1177-1206.

[2] G. Darrieus, Propagation d'un front de flamme, Unpublished work presented at "Le Congres de Mecanique Appliquee," 1938.

[3] L. D. LANdau, On the theory of slow combustion, Acta Physicochimica, USSR, 19 (1944), pp. $77-85$.

[4] D. M. Michelson and G. I. Sivashinsky, Nonlinear analysis of hydrodynamic instability in laminar flames-II. Numerical experiments, Acta Astronautica, 4 (1977), pp. 1207-1221.

[5] D. M. Michelson and G. I. Sivashinsky, Thermal-expansion induced cellular flames, Combust. Flame, 48 (1982), pp. 211-217.

[6] G. Joulin And P. Combray, On a tentative, approximate evolution equation for markedly wrinkled premixed flames, Combust. Sci. and Tech., 81 (1992), pp. 243-256.

[7] O. Rahibe, N. Aubry, And G. I. Sivashinsky, Stability of pole solutions for planar propagating flames, Phys. Rev. E, 54 (1996), pp. 4958-4972.

[8] O. Rahibe, N. Aubry, And G. I. Sivashinsky, Intability of pole solutions for planar propagating flames in sufficiently large domains, Combust. Theory Modelling, 2 (1998), pp. $19-41$.

[9] S. Gutman and G. I. Sivashinsky, The cellular nature of hydrodynamic flame instability, Phys. D, 43 (1990), pp. 129-139.

[10] A. G. Istratov And V. B. Librovich, On the stability of gasdynamic discontinuities associated with chemical reactions; the case of a spherical flame, Astronautica Acta, 14 (1969), pp. 453-467.

[11] E. GROFF, The cellular nature of confined spherical propane-air flames, Combust. Flame, 48 (1982), pp. 51-62.

[12] P. Cambray, K. Joulin, And G. Joulin, Coalescence problems in the theory of expanding wrinkled premixed flames, Combust. Sci. Tech., 112 (1996), pp. 271-299.

[13] O. Kupervasser, Z. Olami, and I. Procaccia, Geometry of developing flame fronts: Analysis with pole decomposition, Phys. Rev. Lett., 76 (1996), pp. 146-149.

[14] Z. Olami, B. Galanti, O. Kupervasser, and I. Procaccia, Random noise and pole dynamics in unstable front propagation, Phys. Rev. E, 55 (1997), pp. 2649-2663.

[15] D. Vaynblat and M. Matalon, Stability of pole solutions for planar propagating flames: II. Properties of eigenvalues/eigenfunctions and implications to stability, SIAM J. Appl. Math., 60 (2000), pp. 703-728.

[16] M. Renardy, A model equation in combustion theory exhibiting an infinite number of secondary bifurcations, Phys. D, 28 (1987), pp. 155-167.

[17] O. Thual, U. Frisch, And M. HÉnOn, Application of pole decomposition to an equation governing the dynamics of wrinkled fronts, J. Physique, 46 (1985), pp. 1485-1494. 
[18] Y. C. Lee And H. H. Chen, Non-linear dynamical models of plasma turbulence, Phys. Scripta, T 2 (1982), pp. 41-47.

[19] W. H. Press, B. P. Flannery, S. A. Teukolsky, and W. T. Vetterling, Numerical Recipes in Fortran 77: The Art of Scientific Computing, Cambridge University Press, Cambridge, UK, 1997.

[20] R. A. Horn and C. A. Johnson, Matrix Analysis, Cambridge University Press, Cambridge, UK, 1985.

[21] B. T. Smith, J. M. Boyle, J. J. Dongarra, B. S. Garbow, Y. Ikebe, V. C. Klema, and C. B. Moler, Matrix Eigensystem Routines-EISPACK Guide, Lecture Notes in Comput. Sci. 6, Springer-Verlag, Heidelberg, 1976. 